\title{
A Three-dimensional Mathematical Model for the Pyrolysis of Wet Wood
}

\author{
R. YUEN, ${ }^{1}$ R. CASEY, G. DE VAHL DAVIS and E. LEONARDI \\ School of Mechanical and Manufacturing Engineering \\ University of New South Wales \\ NSW 2052, Australia
}

G.H. YEOH, V. CHANDRASEKARAN and S.J. GRUBITS

Division of Building, Construction \& Engineering

CSIRO, North Ryde NSW 2113, Australia

\begin{abstract}
A three-dimensional mathematical model for the pyrolysis of wet wood including a detailed consideration of the evaporation of moisture, anisotropic and variable properties, and pressure driven internal convection of gases in wood has been developed. A computational code has been formulated modelling multiple competing reactions for up to six constituents, although a single firstorder Arrhenius reaction is adopted in the results presented. The pyrolysis of a beech wood cube with different initial moisture contents has been computed for a range of furnace temperatures. The Arrhenius kinetic parameters have been determined by a method of best fit to the experimental results of Bonnefoy et al [16]. The values agree very well with the range of kinetic data reported by Tinney [4]. The computed results giving a full 3-D description of the transient chemical and physical processes of the pyrolysis of a wet wooden cube heated in a furnace are presented. The influences of the anisotropic properties due to the grain structure on heat and mass transfers in pyrolysing wood are discussed.
\end{abstract}

Keywords: anisotropy; evaporation; heat transfer; kinetic data; mass transfer; modelling; moisture content; pyrolysis; wood.

\section{INTRODUCTION}

Fires in buildings and their associated hazards have received considerable scientific attention over the past few decades. One of the most common materials used in buildings is wood. To assist in understanding the combustibility and flame spreading behaviour of materials, a three-dimensional (3-D) mathematical model for fire simulation which incorporates wood pyrolysis and gas combustion has been developed $[1,2]$. In order that effective predictions can be made with the fire

${ }^{1}$ Present address: Department of Building and Construction, City University of Hong Kong, Tat Chee Avenue, Kowloon, Hong Kong. 
model, it is essential that the model for wood pyrolysis be validated and be adequately comprehensive to account for the anisotropic, hygroscopic and kinetic properties of wood.

Since the development of a one-dimensional (1-D) mathematical model for wood pyrolysis by Bamford et al [3], experiments using wood samples of various sizes and shapes heated inside furnaces with closely controlled temperatures and non-oxidising environments have been carried out for the determination of kinetic data and for the validation of models. Transient quantities including the mass loss and temperature rise of the samples are usually measured. The 1-D mathematical models for the pyrolysis of dry wood have been employed to estimate the kinetic data of the wood pyrolysis by Tinney [4], Kanury et al [5], Kanury [6] and Kung [7,8]. Roberts [9] reviewed the kinetic data and concluded that they were dependent on the size of the sample. Alves et al [10] proposed further a 1-D model for the pyrolysis of wet wood with six-reaction schemes taking into account the different constituents in wood such as hemicellulose, cellulose and lignin, where the corresponding kinetic data were obtained by method of multistage isothermal thermogravimetry. Fredlund [11,12] and Di Blasi [13] proposed 2-D models with the consideration of pressure driven internal convection of gases in the pyrolysing wood. The former model considered moisture evaporation in wet wood pyrolysis, while the latter considered only dry wood pyrolysis with the primary and secondary reaction schemes following the work of Bradbury et al [14] and Broido et al [15]. In the situation of heating large cubical wood samples in furnaces, both 1-D and 2-D mathematical models for wood pyrolysis cannot precisely represent the boundary conditions. Recently, Bonnefoy et al [16] reported a 3-D model for wood pyrolysis and obtained the kinetic data for beech wood pyrolysis using the experimental mass loss measurements. Their model, however, has not considered the moisture content and the anisotropic nature of wood, nor the convective heat transfer as a result of the internal flows of volatile gases produced in the pyrolysis processes.

In this paper, a 3-D mathematical model for the pyrolysis of wood is presented. The pyrolysis reaction is modelled by a first-order Arrhenius reaction, although six first-order reactions representing the competing thermal degradation reactions of various constituents such as the cellulose, hemicellulose, lignin and other minor constituents have been formulated in the computational code. Evaporation of moisture inside the wood is handled through a consideration of the saturation vapour pressure. An energy equation incorporating heat conduction, internal convection due to movement of the water vapour, volatile and inert gases inside the pyrolysing wood as well as the heats of pyrolysis and evaporation, has been formulated. The transport of gases and vapour through the charring solid is assumed to obey Darcy's Law. Mass conservation equations describing the vapour, volatile and inert gases in wood are satisfied. The anisotropic properties of wood including conductivities and permeabilities due to the structure of the grains in wood have been accounted for. The thermophysical properties of the charring wood has been assigned an extent-of-reaction and porosity dependence. The governing equations were transformed into a non-orthogonal curvilinear coordinate system, making the model applicable to a large range of geometries.

For this current application, the 3-D model had been used to predict the pyrolysis of a wooden cube inside a furnace under various temperatures. The transformed governing equations were solved numerically using a control volume technique. Simulations were performed for the pyrolysis of wooden cubes of different initial moisture contents at furnace temperatures of $973 \mathrm{~K}$ and $1273 \mathrm{~K}$. The activation energy and pre-exponential factor for the pyrolysis reaction of the single constituent have been adjusted to fit the measured mass loss history reported by Bonnefoy et al [16]. Detailed results showing the transient development and distribution of the temperature, pressure, gases flow, moisture evaporation and char fraction for different furnace temperatures are presented. The effects 
of the grain structure and the presence of moisture on the heat and mass transfer in the pyrolysing wood are discussed.

\section{MATHEMATICAL MODEL}

The 1-D mathematical models for wood pyrolysis [5-10] have previously been applied for the determination of kinetic data for limited dimensions of slabs or cylindrical wood samples. However, for cubical wood samples, the pyrolysis, which involves the interactions between the chemical and physical processes in the solid, must be modelled by a 3-D pyrolysis model incorporating the moisture content, anisotropic properties of wood and the internal convection of gases. The presence of moisture in hygroscopic materials such as wood will have a significant effect on their characteristics of ignition and hence affect their combustibility [2].

In the current model, the rate of pyrolysis is described by a first-order Arrhenius reaction:

$$
R_{p}=-\frac{\partial \rho_{a}}{\partial t}=\rho_{a} A_{p} \exp \left[-E_{p} /\left(R_{g} T_{s}\right)\right]
$$

where $\rho_{a}$ is the density of the active volatile portion of wood; $A_{p}$ is the pre-exponential constant; $E_{p}$ is the activation energy for the pyrolysis reaction; $R_{g}=8.3144 \mathrm{~kJ} \mathrm{kmol}^{-1} \mathrm{~K}^{-1}$ is the universal gas constant; and $T_{s}$ is the local solid temperature. Although a single first-order reaction has been used in the simulations presented in this paper, the computational code has been formulated to accommodate multiple reactions accounting for the competing reactions of up to six constituents in the solid.

The heat transfer in the solid occurs by thermal conduction and internal convection. The conservation equation for energy is expressed in a non-orthogonal curvilinear coordinate system $\xi^{i}$ by the following equation:

$$
\begin{aligned}
\sqrt{g} \frac{\partial\left[\left(\rho_{s} C_{p s}+\rho_{\ell} C_{p \ell}\right) \theta_{s}\right]}{\partial t} & =\frac{\partial}{\partial \xi^{i}}\left(\beta_{k i} \beta_{k j} \lambda_{s}^{k} \frac{\partial \theta_{s}}{\partial \xi^{j}}\right)-\frac{\partial\left[\beta_{k i}\left(\dot{m}_{g}^{k} C_{p g}+\dot{m}_{v}^{k} C_{p v}+\dot{m}_{i}^{k} C_{p i}\right) \theta_{s}\right]}{\partial \xi^{i}} \\
& -\sqrt{g}\left(H_{p} R_{p}+H_{e v} R_{e v}\right)
\end{aligned}
$$

where $C_{p}, \Delta H_{p}$ and $\Delta H_{e v}$ are the specific heat capacities, heat of pyrolysis and evaporation, respectively, defined with reference to a datum $T_{r e f} ; \theta_{s}=T_{s}-T_{r e f}$ is the modified solid temperature; $R_{e v}$ is the rate of moisture evaporation. The superscript $k=x, y, z$ denotes the directions along (i.e. parallel) and across (i.e. perpendicular) grains to account for the anisotropic properties of wood. The mass fluxes and densities are denoted by $\dot{m}$ and $\rho$ with the subscripts $s, \ell, g, v$ and $i$ denoting the solid, moisture in liquid phase, vapour, volatile and inert gases. The vapour and inert gases are assumed to be present initially in the wood at ambient conditions. Also, $\lambda_{s}$ represents the thermal conductivity of the charring wood. $\beta_{k i}, \beta_{k j}$ and $\sqrt{g}$ represent the geometric coefficients and the Jacobian of the non-orthogonal curvilinear transformation.

The transfer of the vapour, volatile and inert gases is assumed to obey Darcy's Law, with the transfer coefficient $\alpha_{s}^{k}$ given by the ratio of permeability $D_{s}^{k}$ to kinematic viscosity $v_{s}$ of the mixture. The total mass flux is

$\dot{m}^{k}=-\frac{\alpha_{s}^{k}}{\sqrt{g}} \beta_{k j} \frac{\partial P_{s}}{\partial \xi^{j}}$

where $\alpha_{s}^{k}=D_{s}^{k} / v_{s}$. The kinematic viscosity of the mixture is defined by $v_{s}=\mu_{s} / \rho_{t}$ where $\mu_{s}$ is the dynamic viscosity of the mixture; $\rho_{t}=\rho_{g}+\rho_{i}+\rho_{v}$ is the sum of the densities of vapour, volatile 
and inert gases; and $P_{s}$ is the total pressure given by the sum of the partial pressures of vapour, volatile and inert gases, $P_{s}=P_{g}+P_{i}+P_{v}$. The dynamic viscosity of the mixture is given by the weighted sum of the dynamic viscosities of the gaseous species in wood. Individual mass fluxes of the vapour, volatile and inert gases are obtained by weighted fractions of the total mass flux with respect to the gaseous species densities.

The equations for the conservation of mass (i.e. continuity) of the vapour, volatile and inert gases can be expressed in non-orthogonal curvilinear coordinate system as follows:

$$
\begin{aligned}
& \frac{\partial \rho_{v}}{\partial t}=\frac{1}{\sqrt{g}} \frac{\partial}{\partial \xi^{i}}\left(\frac{\alpha_{s}^{k} \rho_{v}}{\rho_{t}} \beta_{k i} \beta_{k j} \frac{\partial P_{s}}{\partial \xi^{j}}\right)+R_{e v} ; \frac{\partial \rho_{g}}{\partial t}=\frac{1}{\sqrt{g}} \frac{\partial}{\partial \xi^{i}}\left(\frac{\alpha_{s}^{k} \rho_{g}}{\rho_{t}} \beta_{k i} \beta_{k j} \frac{\partial P_{s}}{\partial \xi^{j}}\right)+R_{p} ; \text { and } \\
& \frac{\partial \rho_{i}}{\partial t}=\frac{1}{\sqrt{g}} \frac{\partial}{\partial \xi^{i}}\left(\frac{\alpha_{s}^{k} \rho_{i}}{\rho_{t}} \beta_{k i} \beta_{k j} \frac{\partial P_{s}}{\partial \xi^{j}}\right)
\end{aligned}
$$

The vapour, volatile and inert gases are assumed to obey the ideal gas law. The densities of individual gases are assigned a dependence on porosity.

A combined pressure equation representing the conservation of mass is obtained

$$
\sqrt{g} \frac{\partial\left(\pi_{g} P_{s} / R_{g} T_{s}\right)}{\partial t}=\frac{\partial}{\partial \xi^{i}}\left[\frac{\alpha_{s}^{k}}{\rho_{t}}\left(\frac{\rho_{v}}{M_{\ell}}+\frac{\rho_{g}}{M_{g}}+\frac{\rho_{i}}{M_{i}}\right) \beta_{k i} \beta_{k j} \frac{\partial P_{s}}{\partial \xi^{j}}\right]+\sqrt{g}\left(\frac{R_{e v}}{M_{\ell}}+\frac{R_{p}}{M_{g}}\right)
$$

where $M$ is the molar mass and $\pi_{g}$ is the porosity.

The evaporation of moisture in the wood is assumed to be sufficiently rapid to attain thermodynamic equilibrium, i.e. $P_{v}=P_{v s}$, with the saturation vapour pressure $P_{v s}$ given by

$$
P_{v s}=K_{1} \exp \left(-K_{2} / T_{s}\right)
$$

where $K_{1}$ and $K_{2}$ are constants relevant to the temperature range being studied. The rate of evaporation of moisture is given by

$$
R_{e v}=\frac{\partial \rho_{v}}{\partial t}-\frac{1}{\sqrt{g}} \frac{\partial}{\partial \xi^{i}}\left(\frac{\alpha_{s}^{k} \rho_{v}}{\rho_{t}} \beta_{k i} \beta_{k j} \frac{\partial P_{s}}{\partial \xi^{j}}\right) \text {. }
$$

The thermal conductivity $\lambda_{s}^{k}$ and volumetric heat capacity $\rho_{s} C_{p s}$ of the pyrolysing wood are normally assumed to vary linearly with char fraction from the initial value of the virgin wood to that of the char $[11,13]$

$\lambda_{s}^{k}=a \lambda_{w}^{k}+(1-a) \lambda_{f}^{k} ; \rho_{s} C_{p s}=a \rho_{w} C_{p w}+(1-a) \rho_{f} C_{p f}$

where $\lambda_{w}^{k}, \lambda_{f}^{k}, C_{p w}$ and $C_{p f}$ are the thermal conductivities and specific heat capacities of the virgin wood and final char respectively and $a=\left(\rho_{s}-\rho_{f}\right) /\left(\rho_{w}-\rho_{f}\right)$. The final char density $\rho_{f}$ is assumed to be known a priori.

The permeability of the pyrolysing solid is described by an exponential function of the varying solid density as the wood progressively transforms to char:

$D_{s}^{k}=K_{D 1}^{k} \exp \left(-K_{D 2}^{k} c\right)$

where $c$ is a function of char fraction. $K_{D 1}^{k}$ and $K_{D 2}^{k}$ are constants depending on species and orientation of the wood grains. All other thermodynamic and transport properties vary linearly with temperature. Thermal swelling, shrinkage, surface regression and possible surface oxidation reactions of the virgin wood and char are neglected. 


\section{NUMERICAL METHOD}

The conservation equations were discretised using a control volume technique. The convection was modelled using a hybrid differencing scheme. The discretised equations were solved using the Strongly Implicit Procedure [17]. The 3-D pyrolysis model has been used to simulate pyrolysis of a wooden cube of dimensions $10 \times 10 \times 10$ $\mathrm{mm}$. Taking advantage of the cubical shape, only one-eighth of the cube was considered as the domain for simulation. Figure 1 shows the computational domain with reference to the actual dimension of the cube. A total of $15 \times 15 \times 15$ control volumes in the directions along and across the grains were used. A non-uniform mesh was used in the wood with the mesh concentrated adjacent to the heated external surfaces of the cube, to handle the steep gradients there.

\section{BOUNDARY CONDITIONS}

The wood is heated by convection and radiation from its surroundings. The boundary conditions for the governing energy and pressure equations at the planes of symmetries at $x=5$ $\mathrm{mm}, y=5 \mathrm{~mm}$ and $z=5 \mathrm{~mm}$ are

$\frac{\partial T_{s}}{\partial n}=0 ;$ and $\frac{\partial P_{s}}{\partial n}=0$

and those for the heated external surfaces at $x=0, y=0$ and $z=0$ are

$\lambda_{s}^{n} \frac{\partial T_{s}}{\partial n}=-\varepsilon \sigma\left(T_{s o}^{4}-T_{f}^{4}\right)-h_{c}\left(T_{s o}-T_{g}\right) ; P_{s}=P_{0}$

where $\varepsilon$ denotes the surface emissivity; $\sigma=56.7 \times 10^{-12} \mathrm{kWm}^{-2} \mathrm{~K}^{-4}$ the Stefan-Boltzmann constant; $T_{s o}$ the wood surface temperature; $T_{f}$ the furnace temperature; $T_{g}$ the furnace gas temperature; $P_{o}=1.013$ bar the ambient pressure and $n$ denotes the direction normal to the boundary surface.

\section{OTHER PROPERTY VALUES}

Computations have been performed using the current 3-D pyrolysis model for wooden cubes of $0 \%$ and $9 \%$ initial moisture contents at the furnace temperatures of $973 \mathrm{~K}$ and $1273 \mathrm{~K}$. The initial temperatures of the wood and ambient were assumed to be $25{ }^{\circ} \mathrm{C}$. The emissivity of the surface of the wood was assumed to be 0.85 .

The constants $K_{1}$ and $K_{2}$ for expression of the saturated vapour pressure are $4.143 \times 10^{4} \mathrm{MPa}$ and $4822 \mathrm{~K}$ respectively for the range of temperatures studied. The specific heats were taken to be: $C_{p w}=1400+0.3 \theta_{s}, C_{p f}=700+0.6 \theta_{s}, C_{p \ell}=4190 \mathrm{Jkg}^{-1} \mathrm{~K}^{-1}$; and

$C_{p g}=1000+0.8 \theta_{s}, C_{p i}=1062.9, C_{p v}=1880 \mathrm{Jkg}^{-1} \mathrm{~K}^{-1}$.

For anisotropic properties of wood, the thermal conductivities and permeabilities of the virgin wood and char are given by $[11,13]$

$$
\begin{aligned}
& \lambda_{w}^{x}=0.255, \lambda_{f}^{x}=0.1046 \quad \mathrm{Wm}^{-1} \mathrm{~K}^{-1} ; \text { and } \\
& \lambda_{w}^{y}=\lambda_{w}^{z}=0.105, \lambda_{f}^{y}=\lambda_{f}^{z}=0.071 \quad \mathrm{Wm}^{-1} \mathrm{~K}^{-1}
\end{aligned}
$$


$K_{D 1}^{x}=4.935 \times 10^{-14} \mathrm{~m}^{2}, K_{D 2}^{x}=4.605 ;$ and

$K_{D 1}^{y}=K_{D 1}^{z}=4.935 \times 10^{-18} \mathrm{~m}^{2}, K_{D 2}^{y}=K_{D 2}^{z}=11.513$.

The dynamic viscosities for the gas species in wood were taken to be $\mu_{g}=8.5 \times 10^{-6}+0.0295 \times 10^{-6} \theta_{s} \mathrm{~kg} \mathrm{~m}^{-1} \mathrm{~s}^{-1}$; and

$\mu_{v}=8.5 \times 10^{-6}+0.0375 \times 10^{-6} \theta_{s}$ and $\mu_{i}=3.178 \times 10^{-5} \mathrm{~kg} \mathrm{~m}^{-1} \mathrm{~s}^{-1}$.

The molecular weights for the various gas species in both the solid and gas phase were assumed to be: $M_{g}=75, M_{v}=18$ and $M_{i}=28$.

The heat of pyrolysis $\Delta H_{p}=322 \mathrm{~kJ} \mathrm{~kg}^{-1}$ (endothermic) has been adopted. The heat of evaporation $\Delta H_{e v}$ was taken to be $2260 \mathrm{~kJ} \mathrm{~kg}^{-1}$. The final char density $\rho_{f}$ and the initial wood density $\rho_{w}$ of $91.56 \mathrm{kgm}^{-3}$ and $700 \mathrm{kgm}^{-3}$, respectively, were used.

\section{RESULTS}

To determine the activation energy $E_{p}$ and the pre-exponential factor $A_{p}$, the model has been used to simulate the pyrolysis of a wet wooden cube with an initial moisture content $X_{0}=9 \%$ at furnace temperatures $T_{f}=973 \mathrm{~K}$ and $T_{f}=1273 \mathrm{~K}$ and the computed mass loss history has been compared with the experimental results on beech wood in [16]. Figure 2 shows the best agreement achieved between the computed and experimental mass losses vs. time for the pyrolysis of the wooden cube. The result was obtained using an activation energy $E_{p}=125 \mathrm{~kJ} \mathrm{~mole}^{-1}$ and a preexponential factor $A_{p}=1.25 \times 10^{8} \mathrm{~s}^{-1}$. Excellent agreement is observed in the case of $T_{f}=1273 \mathrm{~K}$. For $T_{f}=973 \mathrm{~K}$, the computed and measured values agree extremely well after about $35 \mathrm{~s}$ until completion of pyrolysis (i.e. during the middle and final stages). In the early stage, however, there was a discrepancy. It is probably due to the use of only a single kinetic reaction for the modelling of the devolatilisation process. It is well known that [14] competing primary reactions due to the different major constituents (i.e. cellulose, hemicellulose and lignin) and secondary reactions exist in wood pyrolysis. As mentioned above, the current computational code has been formulated with multiple competing reactions for the major constituents. The prediction may be improved if these are modelled. The overall agreement is still considered to be very good. The selected values of activation energy and pre-exponential factor are also in good agreement with the values $E_{p}=124.7 \mathrm{~kJ} \mathrm{~mole}^{-1}$ and $A_{p}=6 \times 10^{7}-7.5 \times 10^{8} \mathrm{~s}^{-1}$ reported in [4]. The pre-exponential factor, however, is higher than the value of $5 \times 10^{6} \mathrm{~s}^{-1}$ reported by Bonnefoy et al [16]. This is probably

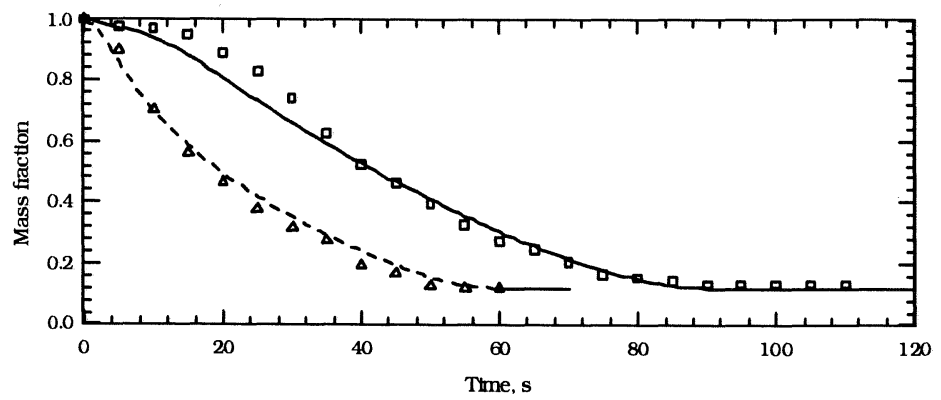

FIGURE 2. The computed mass fraction loss at the furnace temperature of $973 \mathrm{~K}(\rightarrow)$ and $1273 \mathrm{~K}(--)$, compared with experimental values at $973 \mathrm{~K}(\square)$ and $1273 \mathrm{~K}(\Delta)$ reported in [16]. 
due to the absence of the moisture evaporation in their 3-D model. As the presence of the evaporation processes appears to slow down the initial process of temperature rise in wood, a higher value of pre-exponential factor is expected in the current model which includes the consideration of moisture evaporation.

A set of the computed transient results for the wet wood of $X_{0}=9 \%$ heated at a furnace temperature of $973 \mathrm{~K}$ are presented as an example and discussed in this paper.

Figure 3 shows the isotherms and char fraction $\left(\rho_{c} / \rho_{w}\right)$ and moisture content contours in the cube at $t=10 \mathrm{~s}$ for the furnace temperature of $973 \mathrm{~K}$, on the $y-z$ plane at $x=5 \mathrm{~mm}$ (mid-plane) and

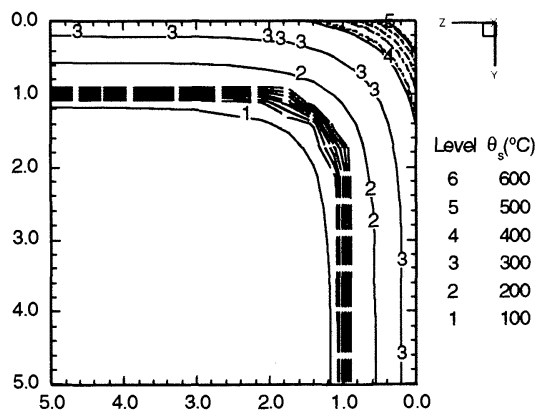

FIGURE 3. Isotherms (-) from 100 to $600{ }^{\circ} \mathrm{C}$, char fraction contours (- - ) from 0.02 to 0.1 with step 0.02 and moisture content contours (- ) from $2 \%$ to $7 \%$ with step $1 \%$ on $y-z$ plane at $x=5 \mathrm{~mm}$ (mid-plane) at $t$ $=10 \mathrm{~s}$ for $T_{f}=973 \mathrm{~K}$ and $X_{0}=9 \%$.

perpendicular to the direction of the grains. The temperature gradients as indicated by the isotherms are identical along the $y$ and $z$ axis on the plane. This is expected due to the similar thermal conductivity and permeability in both directions which are perpendicular (i.e. across) to the grains. The char fraction and moisture content contours are found to exhibit similar behaviour. The char fraction contour of 0.02 (innermost) gives a good indication of the char front. The region bounded by the char fraction contours of 0.02 and 0.1 represents the zone of active devolatilisation. The moisture content contours also indicate the advancement of the evaporation process in wood. The region enclosed by the moisture content contours of $2 \%$ (outermost) and $7 \%$ (innermost) represents the zone of evaporation.

Figure 4 consists of a set of four plots showing the total gas pressure contours and the total mass flux vector field at $10,20,30$ and $40 \mathrm{~s}$ for the furnace temperature of $973 \mathrm{~K}$, on the $x-z$ plane at $y=5 \mathrm{~mm}$ (mid-plane) and cutting parallel to the direction of grains. The effect of the anisotropic properties of wood on the pyrolysis can be best demonstrated by the plots of transient flows and pressure contours.

A complicated system of pressure and gas flows is observed. There are three regions identified. At $t=10 \mathrm{~s}$, the region near to the left lower corner corresponds to the region of virgin wet wood which is neither evaporating nor pyrolysing. This zone consists of primarily the saturated vapour and inert gases which exist initially in the wood. In this zone, the pressure is below 1.05 bar but slightly higher than atmospheric pressure and is rising. This is caused by the slow but gradually increasing temperature as well as the poor permeability of the virgin wood.

Adjacent to this, a narrow region of high pressure is found. The elongated shape of the region with a steep pressure gradient across the grains is due to the much lower permeabilities of wood (i.e. 10000 times less) in this direction than that along the grains. The highest value of the computed pressure is around 2.2 bar, which is in the same order of magnitude as the experimental value of 2.35 bar reported in [11]. This represents a region of rapid evaporation of the moisture in the wood.

A third region of high pressure, around $1.15 \mathrm{bar}$, is found very close to the right hand surface $(z=0)$. This corresponds to the onset of the devolatilisation after the drying of the wood. The pressure rise is caused by the volatile gas produced when the permeability is still low. The order of magnitude of the pressure in this region is comparable to the experimental value of 1.14 bar for the pyrolysis of dry wood [11]. 


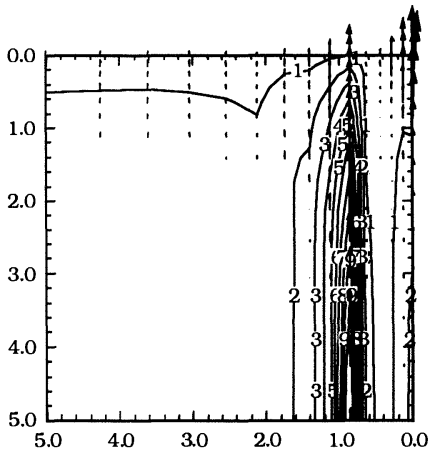

(a)

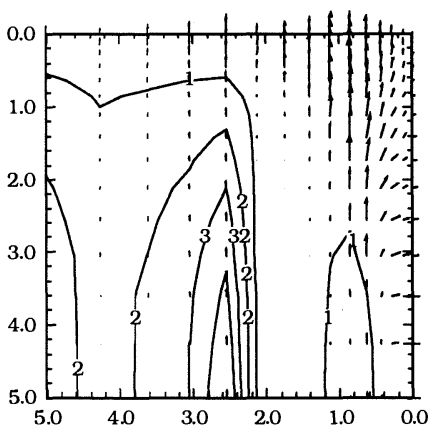

(c)

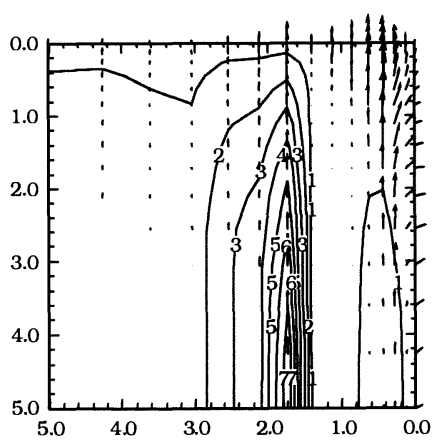

(b)

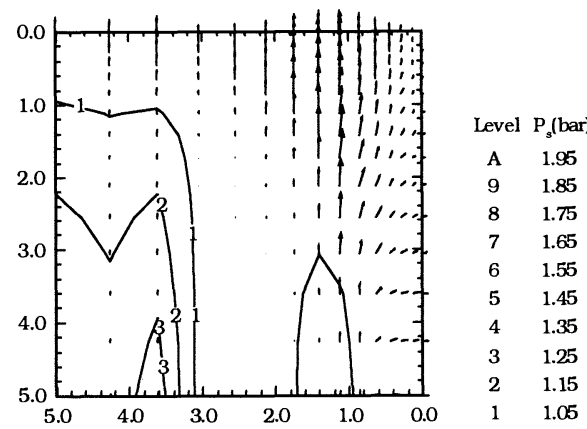

(d)

FIGURE 4. Contours of total gas pressure and total mass flux vector field on $x-z$ plane at $y=5 \mathrm{~mm}$ (mid-plane) at (a) $t$ $=10 \mathrm{~s}$, (b) $t=20 \mathrm{~s}$, (c) $t=30 \mathrm{~s}$ and (d) $t=40 \mathrm{~s}$ for $T_{f}=973 \mathrm{~K}$ and $X_{0}=9 \%$.

From figure 4 (b) to (d), as the heating continues, evaporation starts to occur in the inner part which results in the migration of the high pressure zone towards the centre of the cube. The devolatilisation zone is also found to move inwards following the evaporation zone. There is a tendency for a decrease of the peak pressure and pressure gradients in these two zones as the charred area expands near the top $(x=0)$ and right hand surfaces $(z=0)$ over time.

Total mass flux vectors indicating the magnitude and directions of flows of gases and vapour are plotted in figure 4 . The flow of gases and vapour are found to be governed strongly by the grain structure with significant movement along the grains except near the surface $z=0$.

At the early stage around 20 s, while the devolatilisation is still concentrated at the right hand surface $(z=0)$, a considerable outflow of gases is found also at that surface. As the char front advances further into the wood and the charred area expands, the outflow of gases at the right hand surface becomes less significant.

Figure 4 shows two streams of gases leaving the top surface $(x=0)$ which represent the outflow of the gases produced by the evaporation and devolatilisation. The stream above the pressure zone due to devolatilisation as discussed above consists mainly of volatile gases. The other stream which follows closely the movement of the pressure zone due to evaporation corresponds mainly to the outflow of vapour. It is found to disappear after the evaporation completes around 40s. 
Figure 5 (a) to (d) show the total gas pressure contours and the total mass flux vector field in the cube at 50,60,70 and $80 \mathrm{~s}$ for the furnace temperature of $973 \mathrm{~K}$, on the $x-z$ plane at $y=0 \mathrm{~mm}$ (mid-plane). Unlike figure 4 above, due to the completion of the evaporation in wood, only one zone with moderately high pressure is observed in each of the plots. This corresponds to the build up of pressure due to the production of the volatile gases. Steeper pressure gradients are found in directions across the grains indicating again the effects of the structure of grain in wood on the internal flow of the gases in wood. Following the advancement of the isotherms towards the centre (see figure 6), the pressure region moves gradually towards the mid-plane $(z=5 \mathrm{~mm})$.

As shown by the mass flux vectors in the figure 5, most of the gas leaves the cube at the top surface $(x=0)$ due to the grain orientation. In time, with the formation of a large area of char near the top surface, there is an increase of outflow of gases from the top surface. The position of the maximum outflow follows closely the lateral movement of the high pressure region and eventually reaches the mid-plane $(z=5 \mathrm{~mm})$.

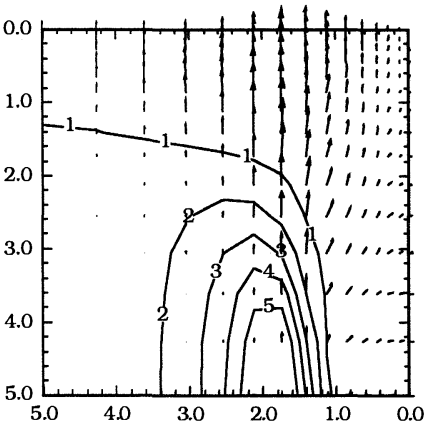

(a)

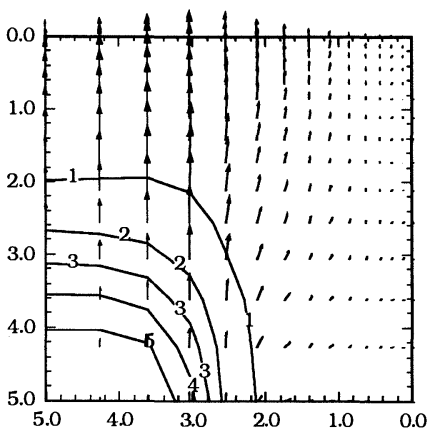

(c)

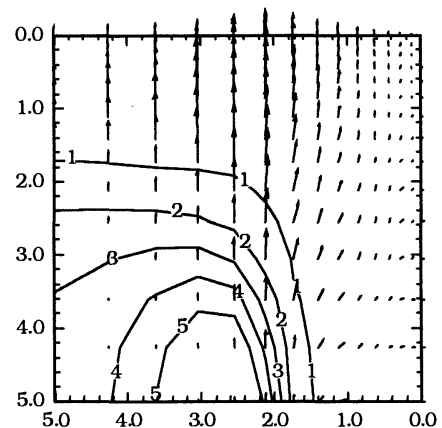

(b)

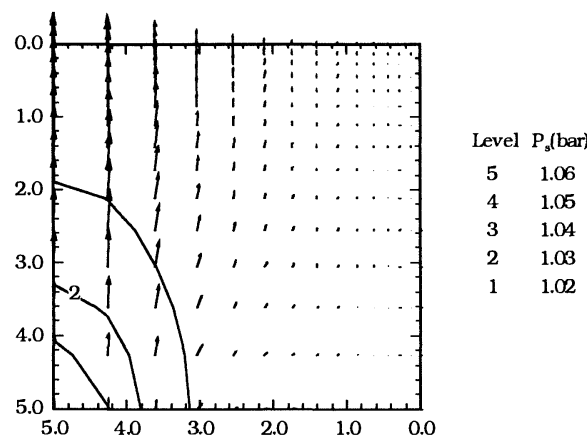

(d)

FIGURE 5. Contours of total gas pressure and total mass flux vector field on $x$-z plane at $y=5 \mathrm{~mm}$ (mid-plane) at (a) $t$ $=50 \mathrm{~s}$, (b) $t=60 \mathrm{~s}$, (c) $t=70 \mathrm{~s}$ and (d) $t=80 \mathrm{~s}$ for $T_{f}=973 \mathrm{~K}$ and $X_{0}=9 \%$.

Figure 6 shows a plot of isotherms together with the char fraction and moisture content contours in the cube at $10,30,60$ and $80 \mathrm{~s}$ for the furnace temperature of $973 \mathrm{~K}$, on the $x-z$ plane at $y=5 \mathrm{~mm}$ (mid-plane). A wave-like propagation is observed for the isotherms from the external surfaces towards the centre of the cube as heating continues. The temperature gradients are weaker 
in the direction along the grains than across due to the relatively higher thermal conductivities in the former direction. On the other hand, the rate of advancement of the isotherms is found to be slower in the direction along the grains. This is due to the presence of large convective cooling as a result of internal flow of vapour and volatile gases in this direction, as reported previously [13]. It is also found in this study that the evaporative cooling due to the faster and earlier evaporation of moisture along the grains as discussed below serves to enhance this phenomenon.

The contours of moisture content indicate an advancing zone of evaporation. The innermost contour represents a moisture content of $7 \%$ and the outermost $1 \%$. Similar to the isotherms, the wave-like propagation is also found with the moisture content contours. The evaporation zone clearly separates the wet region where the evaporation has not yet begun from the dry region where the evaporation has finished.

As expected, the evaporation occurs generally around the temperature of $100{ }^{\circ} \mathrm{C}$. It is interesting to note in figure 6 (a) and (b) that in the direction along the grains, the contour of $7 \%$ moisture content which represents the onset of evaporation (i.e. evaporation front) always moves ahead of the $100^{\circ} \mathrm{C}$ isotherm. In the direction across the grains, however, the evaporation front is always behind the $100{ }^{\circ} \mathrm{C}$ isotherm. This implies that in direction along the grains, the evaporation begins at temperatures slightly lower than $100{ }^{\circ} \mathrm{C}$, while in the direction across the grains, the

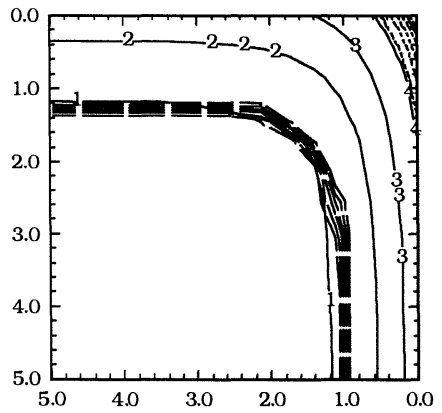

(a)

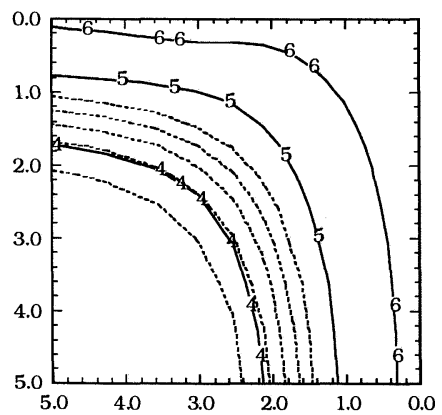

(c)

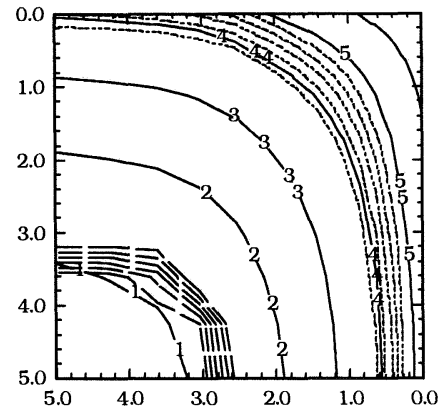

(b)

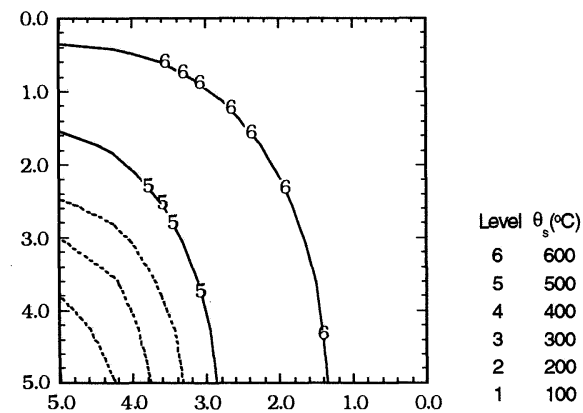

(d)

FIGURE 6. Isotherms $(-)$ from 100 to $600{ }^{\circ} \mathrm{C}$, char fraction contours (- - ) from 0.02 to 0.1 with step 0.02 and moisture content contours (- ) from $2 \%$ to $7 \%$ with step $1 \%$ on $x-z$ plane at $y=5 \mathrm{~mm}$ (mid-plane) at (a) $t=10 \mathrm{~s}$, (b) $t=30 \mathrm{~s}$, (c) $t=60 \mathrm{~s}$ and (d) $t=80 \mathrm{~s}$ for $T_{f}=973 \mathrm{~K}$ and $X_{0}=9 \%$. 
evaporation begins at temperature slightly above $100{ }^{\circ} \mathrm{C}$. This faster and earlier evaporation of moisture can be explained by the higher permeability in this direction along the grains which allows the quicker release of vapour. Consequently, a faster evaporation rate is needed to provide sufficient vapour to maintain saturation inside the porous medium. Also, it appears from the above discussion that this actually assists in cooling the pyrolysing solid. It is therefore inferred that the rate of evaporation of moisture in wood is affected also by its permeability and hence by the grain structure.

At $40 \mathrm{~s}$, as shown in figure 6 (b), the evaporation zone approaches the centre of the cube leaving behind a large region of dry wood. Beyond $50 \mathrm{~s}$, the wood is completely dry.

In figure 6, the contours of char fraction from 0.02 (around $15 \%$ char) to 0.10 (around $85 \%$ char) indicate a region of active devolatilisation in the dry wood region. Similar to the isotherms, the wave-like behaviour is again observed with the char fraction contour of 0.02 representing the char front which marches gradually towards the centre of the cube. Since the devolatilisation process is temperature dependent according to the Arrhenius expression, the devolatilisation zone follows closely the advancement of the isotherms of $400{ }^{\circ} \mathrm{C}$ and $500{ }^{\circ} \mathrm{C}$ throughout the heating process. Also, the region of devolatilisation stretches in time, as the temperature gradients becomes lower. At 80 s, the devolatilisation zone reaches the centre of the cube and the pyrolysis is complete around 90s.

\section{CONCLUSION}

A 3-D mathematical model for the pyrolysis of wet wood has been developed which includes detailed considerations of the evaporation of moisture, anisotropic and variable properties, and pressure driven internal convection of gases in wood. Although a single first-order Arrhenius reaction is modelled, multiple competing reactions for up to six constituents has been formulated in the computational code. The governing equations have been transformed into a non-orthogonal curvilinear coordinate system, making the model applicable to a large range of geometries.

The system of governing equations has been solved numerically using a control volume technique to predict the pyrolysis of a beech wood cube with different initial moisture contents heated at various furnace temperatures. The activation energy of $125 \mathrm{kJmole}^{-1}$ and pre-exponential factor of $1.25 \times 10^{8} \mathrm{~s}^{-1}$ for the pyrolysis reaction of the single constituent has been determined by achieving a best fit to the measured mass loss history reported by Bonnefoy et al [16]. The values agree very well with the range of kinetic data previously reported [4]. The pre-exponential factor, however, is higher than the reported value in [16] due to the presence of moisture evaporation in the current model. This demonstrates the need for a detailed consideration of moisture evaporation for a precise representation of the pyrolysis of hygroscopic material such as wood.

A set of computed results for the pyrolysis of a wet wooden cube with an initial moisture of $9 \%$ heated at a furnace temperature of $973 \mathrm{~K}$ giving a 3-D quantitative description of the transient processes has been selected and presented in this paper. The transient development and distribution of the temperature, pressure, gas and vapour flow, moisture evaporation and char fraction for different furnace temperatures are reported. The influences of the anisotropic properties due to the grain structure on the heat and mass transfers in pyrolysing wood are discussed. An important effect is the faster and earlier evaporation of moisture in the direction along the grains due to the higher permeabilities along the grains. On the other hand, the rate of heating in this direction is slower due to the convective cooling by vapour and volatile gases and to evaporative cooling.

It has been demonstrated in this paper that the current 3-D model for pyrolysis of wet wood can be used to describe the sophisticated chemical and physical processes involved in wood pyrolysis. As the computational code has been formulated to include multiple competing reactions for the 
major constituents, improvements can be made on the predictions by making use of this feature in future.

\section{ACKNOWLEDGEMENTS}

R. Yuen acknowledges with thanks the financial support of the CSIRO Division of Building, Construction and Engineering.

\section{REFERENCES}

1. Yuen, R., Yeoh, G. H., Casey, R., Chandrasekaran, V., de Vahl Davis, G., Grubits, S. and Leonardi, E., "Numerical modelling of the burning of wood in a cone calorimeter", Proceedings of the Ninth International Conference on Numerical Methods for Thermal Problems, Vol. IX, Part 2: 651-662, 1995.

2. Yuen, R., Yeoh, G. H., Casey, R., Chandrasekaran, V., de Vahl Davis, G., Grubits, S. and Leonardi, E., "Prediction of piloted ignition and combustion of wood", Proceedings of Symposium on Thermal Science and Engineering in Honor of Chancellor Chang-Lin Tien, San Francisco, 1995.

3. Bamford, C. H., Crank, J. and Malan, D. H., 'The Combustion of Wood: Part I', Proc. Camb. Phil. Soc., 42: 166-182, 1946.

4. Tinney, E. R., "The combustion of wooden dowels in heated air", Tenth Symposium (International) on Combustion, The Combustion Institute: 925-930, 1965.

5. Kanury, A. M. and Blackshear, JR, P. L., "Some considerations pertaining to the problem of wood-burning", Combustion Science and Technology, 1:339-355, 1970.

6. Kanury, A. M., "Thermal decomposition kinetics of wood pyrolysis", Combustion and Flame, 18: 75-83, 1972.

7. Kung, H. C., "A Mathematical Model of Wood Pyrolysis", Combustion and Flame, 18: 185$195,1972$.

8. Kung, H. C. and Kalelkar, A. S., "On the heat of reaction in wood pyrolysis", Combustion and Flame, 20: 91-103, 1973.

9. Roberts, A. F., "A review of kinetics data for the pyrolysis of wood and related substances", Combustion and Flame, 14: 261-272, 1970.

10. Alves, S. S. and Figueiredo, J. L., "A model for pyrolysis of wet wood", Chemical Engineering Science, 44: 2861-2869, 1989.

11. Fredlund, B., "A model for heat and mass transfer in timber structures during fire, A theoretical, numerical and experimental Study", Report LUTVDG/(TVBB-1003), Lund University, 1988.

12. Fredlund, B, "Modelling of heat and mass transfers in wood structures during fire", Fire Safety Journal, 20: 39-69, 1993.

13. Di Blasi, C., "On the influence of physical processes on the transient pyrolysis of cellulosic samples", International Symposium on Fire Safety Science: 229-240, 1994.

14. Bradbury, A. G. W., Sakai, Y. and Shafizadeh, F., "A kinetic model for pyrolysis of cellulose", Journal of Applied Polymer Science, 23: 3271-3280, 1979.

15. Broido, A. and Nelson, M. A., "Char yield on pyrolysis of cellulose", Combustion and Flame, 24: 263-268, 1975.

16. Bonnefoy, F., Gilot, P. and Prado, G., "A three-dimensional model for the determination of kinetic data from the pyrolysis of beech wood", Journal of Analytical and Applied Pyrolysis, 25: 387-394, 1993.

17. Stone, H. L., "Iterative solution of implicit approximations of multidimensional partial differential equations", SIAM Journal on Numerical Analysis, 5: 530-558, 1968. 